\title{
Postoperative Results of Simple Myringoplasty with Fibrin Glue and Patients' Satisfaction
}

\author{
Hiroshi Inui ${ }^{1)}$ and Takashi Inui ${ }^{2)}$
}

\begin{abstract}
The postoperative results for the simple myringoplasty with fibrin glue (Yuasa method) performed as a day surgery under local anesthesia between August 2000 and December 2012 were analyzed in 82 cases (75 cases of chronic otitis media, 4 cases of traumatic tympanic membrane perforation and others). The mean operating time was 37.4 minutes. The total rate of closure was $89.0 \%$, and the total rate of successful hearing improvement was $91.5 \%$. The perforations were classified into 4 groups. The rates of closure and successful hearing improvement were dropped in patients with large tympanic membrane perforation. In 4 patients with hearing aids who experienced the tympanic membrane perforations, the Yuasa method was performed to reduce the influence of conductive hearing loss. The patients' subjective hearing levels all improved. Furthermore, for 32 cases who underwent the Yuasa method between January 2007 and December 2012, the patient satisfaction was examined using a questionary survey. During the postoperative stage, none of the patients experienced anxiety after their going home.
\end{abstract}

Keywords : postoperative results, simple myringoplasty with fibrin glue, satisfaction, rate of closure, successful hearing improvement

\section{References}

1）湯浅 涼, 西條 茂, 冨岡幸子, 他 : 簡易な鼓膜形成術一 フィブリン糊を用いた接着法一. 耳鼻・頭頸外科 61：1117$1122,1989$.

2) Inui $H$ and Inui $T$ : Status and satisfaction in clinic day surgery. Pract Otol (Kyoto) 103: 865-871, 2010.

3) 日本耳科学会用語委員会：聴力改善の成績判定について (2000 年). Otol Jpn 11: 62-63, 2001.

4) Hayashi K, Oishi M and Shinkawa A : Investigation into the safety and certainty of day surgery on the ear in our clinic. Pract Otol (Kyoto) 100: 525-531, 2007.

5) Kobayashi $Y$ : Day and short stay surgery at our clinic. Pract Otol (Kyoto) 102: 495-501, 2009.

6) Isono M, Fukuyama $Y$ and Murata $\mathrm{K}$ : Tympanic membrane perforations closed with fibrin glue. Pract Otol (Kyoto) 86: 653-662, 1993.

7）小川浩司：外傷性鼓膜穿孔. JOHNS 19：1417-1420, 2003.

8) Inafuku $S$ and Nakayama $M$ : Management of surgical failure cases after tympanoplasty with fibrin glue. Pract Otol (Kyoto) 96: 746-747, 2003.

9）湯浅 涼：耳疾患と短期滞在手術 慢性中耳炎. JOHNS 24: 1145-1149, 2008.

10）安田知久, 中川尚志: 穿孔縁より筋膜を挿入する鼓膜形成 術一Interlay 法の手技と手術成績一. Otol Jpn 13：613-617, 2003.

11）金子明弘，宗本由美，土井 直，他：接着法を用いた鼓膜 形成術の成績と工夫. Otol Jpn 16: 81-86, 2006.

12）柴崎 修, 中嶋正人, 高橋壽彦, 他 : 自己フィブリン糊を 用いた鼓膜形成術（接着法）の術後成績. Otol Jpn 19:654659, 2009.

13）橋本茂久, 山本 裕, 森田由香, 他: 当科における接着法 による鼓膜形成術の検討. Otol Jpn 17: 124-127, 2007.

14）前田 学, 斉藤龍介, 中川文夫, 他：鼓膜形成術における 従来法と接着法の比較. 日耳鼻会報 101：1062-1068, 1998.

15) Hasegawa $\mathrm{Y}$, Yamamoto E, Oiki H, et al. : Postoperative results of type I tympanoplasty. Pract Otol (Kyoto) 105: 737740, 2012 .
1) Inui ENT Clinic

2) ENT Inui Clinic
Corresponding Author Address : Hiroshi Inui inui-ent@xg8.so-net.ne.jp 


\begin{tabular}{ccccc}
\hline & & & \\
\hline \\
\end{tabular}

Classification of tympanic membrane perforations

The perforations were classified into 4 groups. The number of cases, the operative side and other details can be seen.

Postoperative results

The total rate of closure was $89.0 \%$ and the total successful rate of hearing improvement was $91.5 \%$.

\begin{tabular}{c|c|c|c|c|c|c|c|c|c|c}
\hline \hline & \multicolumn{2}{|c|}{ All cases } & \multicolumn{2}{|c|}{ Ia } & \multicolumn{2}{c|}{ Ib } & \multicolumn{2}{c|}{ II } & \multicolumn{2}{c}{ III } \\
\hline Audiogram (mean (dB)) & Pre ope. & Post ope. & Pre ope. & Post ope. & Pre ope. & Post ope. & Pre ope. & Post ope. & Pre ope. & Post ope. \\
\hline Average hearing level & 45.0 & 31.1 & 40.4 & 26.5 & 45.8 & 32.4 & 46.2 & 30.9 & 55.3 & 41.4 \\
\hline Air bone gap & 20.4 & 7.4 & 18.0 & 5.3 & 20.8 & 8.2 & 21.6 & 7.6 & 23.7 & 11.1 \\
\hline Patch gain & \multicolumn{2}{|c|}{13.9} & \multicolumn{2}{|c|}{12.9} & \multicolumn{2}{|c|}{13.9} & \multicolumn{2}{|c|}{15.9} & 12.2 \\
\hline Hearing improvement & \multicolumn{2}{|c|}{14.2} & \multicolumn{2}{|c|}{13.9} & \multicolumn{2}{|c|}{14.2} & \multicolumn{2}{|c|}{15.3} & 14.0 \\
\hline
\end{tabular}

Rate of closure (\%)

\begin{tabular}{l|c|c|c|c|c} 
Perforation none & $73(89.0)$ & $22(91.7)$ & $22(91.7)$ & $23(88.5)$ & $6(75.0)$ \\
\hline Perforation partially & $5(6.1)$ & $2(8.3)$ & 0 & $1(3.8)$ & $2(25.0)$ \\
\hline Re-perforation & $4(4.9)$ & 0 & $2(8.3)$ & $2(7.7)$ & 0 \\
\hline
\end{tabular}

Rate of successful hearing improvement (\%)

\begin{tabular}{l|c|c|c|c|c} 
Success cases & $75 / 82(91.5)$ & $24 / 24(100)$ & $21 / 24(87.5)$ & $24 / 26(92.3)$ & $6 / 8(75.0)$ \\
\hline Hearing gain $\geqq 15 \mathrm{~dB}$ & $35 / 82(42.7)$ & $8 / 24(33.3)$ & $9 / 24(37.5)$ & $13 / 26(50.0)$ & $5 / 8(62.5)$ \\
\hline Hearing level $\leqq 30 \mathrm{~dB}$ & $44 / 82(53.7)$ & $16 / 24(66.7)$ & $10 / 24(41.7)$ & $16 / 26(61.5)$ & $2 / 8(25.0)$ \\
\hline $\begin{array}{l}\text { Post ope. air bone gap } \\
\leqq 15 \mathrm{~dB}\end{array}$ & $74 / 82(90.2)$ & $24 / 24(100)$ & $21 / 24(87.5)$ & $23 / 26(88.5)$ & $6 / 8(75.0)$
\end{tabular}

